\title{
e-Bridge 3.0: A Strategic Approach to Structural Health Monitoring of Bridges in Costa Rica
}

\author{
Cesar Garita ${ }^{1(\bowtie)}$ and Giannina Ortiz ${ }^{2}$ \\ ${ }^{1}$ School of Computer Science, Costa Rica Institute of Technology, Cartago, Costa Rica \\ cesaraitcr.ac.cr \\ ${ }^{2}$ School of Construction Engineering, Costa Rica Institute of Technology, \\ Cartago 159-7050, Costa Rica \\ gortizaitcr.ac.cr
}

\begin{abstract}
The general condition of road infrastructure is a major weakness of the Costa Rican economy. In particular, a significant percentage of national bridges show an average or critical condition regarding parts of their structure. On the other hand, road and bridge infrastructure is crucial for the national economy since it promotes activities such as tourism and commercial trade. In this context, proper planning and prioritization of infrastructure projects is of high importance for related government institutions. In order to support these strategic activities, it is necessary to gather and monitor up-to-date information originating from different distributed systems and tools. e-Bridge 3.0 is a recent on-going project at the Costa Rica Institute of Technology (TEC) aimed at the design of a bridge monitoring system to integrate strategic information about bridge structures. Modern business intelligence techniques will be applied to generate strategic performance indicators regarding for instance general reliability and remaining lifespan. This paper introduces the e-Bridge 3.0 project as an initiative towards the establishment of a national bridge monitoring system, which would have a significant impact on the effectiveness of national civil infrastructure management.
\end{abstract}

Keywords: ICT and infrastructure $\cdot$ Bridges $\cdot$ Structural Health Monitoring

\section{Introduction}

Bridges represent one of the most critical elements of road infrastructure. They are of paramount importance for the general quality of life of individuals and for economic activities such as tourism, goods transportation and businesses. In the particular case of Costa Rica, most bridge structures were built more than 30 years ago and investment on proper maintenance has been minimal. Currently, a national bridge inventory is being carried out by the e-Bridge program at the Costa Rica Institute of Technology (TEC) in conjunction with the National Road Council. Preliminary inspection results indicate that a significant percentage of bridges show a regular or critical condition regarding specific parts of their structure. The primary causes of this situation are varied in nature including scour, vehicle impact, earthquakes, corrosion, as well as lack of adequate monitoring and maintenance activities. 
In this context, the e-Bridge 3.0 - Bridge Monitoring System project aims at the design and development of a system prototype to support the integration of information about national bridge structures regarding technical specifications, structural reliability, performance variables, as well as geographical and environmental aspects. One of the main novel aspects of e-Bridge 3.0 is the application of business intelligence models and tools to generate control panels of strategic key performance indicators of bridges in the country. Such monitoring system will be useful to support strategic maintenance, planning, and optimization of resource investments for related government institutions.

This paper presents the e-Bridge 3.0 project as an on-going multidisciplinary research and development initiative towards the establishment of a bridge monitoring system in Costa Rica. Such system would play a significant role improving the future infrastructure and socio-economic development of the country.

\section{Background and Related Work}

The e-Bridge 3.0 project represents the third stage of a route map established by a research group on bridge health monitoring at TEC. This research group was created in 2011 with the collaboration of several schools including: Construction Engineering, Computer Science, Electronics, Industrial Production Engineering and Forest Engineering. The project is also part of an e-Science Program and holds strong collaborations with related end-user organizations including Costa Rica Ministry of Transportation, National Road Council and local government units. The general aim of the research group is to generate ICT tools to analyze the condition and behavior of bridge structures in order to better support planning and maintenance activities. The application of these tools will contribute to strengthen one of the key pillars of national economic competiveness. Previous stages of e-Bridge covered specific projects with focus on capacity building and information integration (see for instance [1-3]). Following the established road map, after this third stage, we plan to create a spin-off company specializing on Structural Health Monitoring (SHM) services with a Central American scope.

Regarding existing projects and initiatives related to bridge monitoring systems, several reports can be found that survey several available tools and platforms [4-6]. Some of the most comprehensive proprietary systems include BRIMOS and SHM Live. BRIMOS (Bridge Monitoring System) offers a method for damage detection in bridges and other civil structures based mostly on vibration monitoring [7]. BRIMOS supports different kinds of monitoring applications including hot-spot, permanent and cable monitoring. SHM Live is a web site that manages and displays data about structures monitored in real time anywhere in the world [8]. The system allows real-time configuration of event alarms and offers different data visualization options.

Furthermore, there are several systems specializing in SHM data management which are aimed ad storing, retrieving and sharing large amounts of data gathered through monitoring activities [9, 10]. In [11], several examples of data mining operations are providing in order to reach a better understanding of SHM data. Key Performance Indicators (KPIs) associated to bridge SHM have been defined in the 
context of the BRIMOS project, including integrity, operability, fatigue assessment, damage location and life-cycle curve [7].

Considering existing approaches, one of the main differentiating aspects considered for e-Bridge 3.0 is the incorporation of specialized business intelligence techniques and platforms that support data cubes, strategic key performance indicators and dashboards associated to bridge monitoring. Moreover, the system will apply a scientific workflow management approach for data integration from different systems following virtual enterprise / collaborative networks concepts and models (see [2, 12-14]), and it will be tailored to a specific bridge inspection methodology for the national context.

\section{The e-Bridge 3.0 Project}

This section describes the general and specific objectives of the project and outlines the ICT development methodology for the system prototype.

\subsection{Project Objectives and General Architecture}

The general objective of the e-Bridge 3.0 project (2016-2017) is to design a prototype of a bridge health monitoring system including assessment methodologies, environmental risk analysis, diagnostic features and business intelligence techniques to support bridge performance analysis.

More specific objectives include the following points:

- Analyze the information management requirements for the design of the bridge monitoring system.

- Design proper instrumentation protocols for remote electronic bridge monitoring.

- Develop methodologies for the diagnostic of the structural condition of bridges.

- Develop methodologies to assess environmental vulnerability of bridges.

- Design and develop a prototype of a business intelligence system to support decision making in relation to structural health monitoring of bridges.

The main components of e-Bridge 3.0 architecture are based on the architecture defined for e-Bridge 2.0 (see [1] for details). In summary, these components include: a technical information system for bridge structures; sensors systems to measure actual bridge performance; a geoportal to analyze geographical aspects; and an information integration system using scientific workflow management [2]. In e-Bridge 3.0, the information integration system will be expanded to include business intelligence capabilities for the bridge monitoring system.

\subsection{General ICT Development Methodology}

As mentioned earlier, the main output of the project will be a prototype for a bridge monitoring system based on business intelligence techniques. In general, the development of the software prototype will follow a cascade-like model including phases for requirement analysis, design, and development as described in the following paragraphs. 
For the information management requirements analysis of this prototype, potential sponsors and end-users will be identified. Subsequently, user research techniques will be applied and use-case specifications will be delivered describing the main system processes and user interactions.

For the design of the business intelligence component, a thorough evaluation and selection of available technologies will be carried out based on carefully defined criteria according to the project characteristics. Strategic performance indicators will be integrated in a centralized database from different system components or information sources. Potential strategic indicators associated to bridge information may include for instance: reliability index, global technical assessment, remaining life time, environmental risks, socio-economic importance, and structural damage, among others.

The integration of these indicators from different components will be implemented with web services and scientific workflows. Once the indicators associated to related information sources are processed and stored in the database, the business intelligence prototype will allow strategic queries through data cubes and dashboards using tools such as Pentaho and available extensions. For the prototype development, free and opensource technologies will be preferred whenever possible.

Finally, the project follows a detailed action plan including dissemination activities such as academic publications and the organization of national workshops on SHM.

\section{Preliminary Results}

Using preliminary technical data from visual inspections of around 150 bridges carried by the e-Bridge team, an initial data cube (multi-dimensional data structure for rapid query analysis) has been designed and tested using Pentaho [15]. Current cube dimensions include bridges, regions, evaluations, and routes, so that queries regarding different variables can be solved. In this way, data cube analysis can provide answers to relevant questions such as:

- Average bridge length by type of structure and province.

- Amount of bridges per route.

- Amount of bridges per province.

These queries can be easily solved using built-in drag-and-drop functionalities of Pentaho based on fields associated to predefined data cube dimensions and measures.

The results of the queries can be displayed in a wide variety of graphical possibilities. For instance, Fig. 1 shows the graphical result for a query regarding the average length of bridges by province and type of structure. Namely, the horizontal axis represents provinces and for each province, bars of different colors indicate the average bridge length (vertical axis) per type of structure e.g. culvert, truss, continuous beam.

These charts will be integrated as strategic indicators dashboards to monitor and query different aspects of national bridges from a web-based application. The userfriendly execution of queries and the flexible visualization of the results have proven to be extremely valuable features for the construction engineers responsible for carrying out the bridge inspections and for generating the associated official reports. 


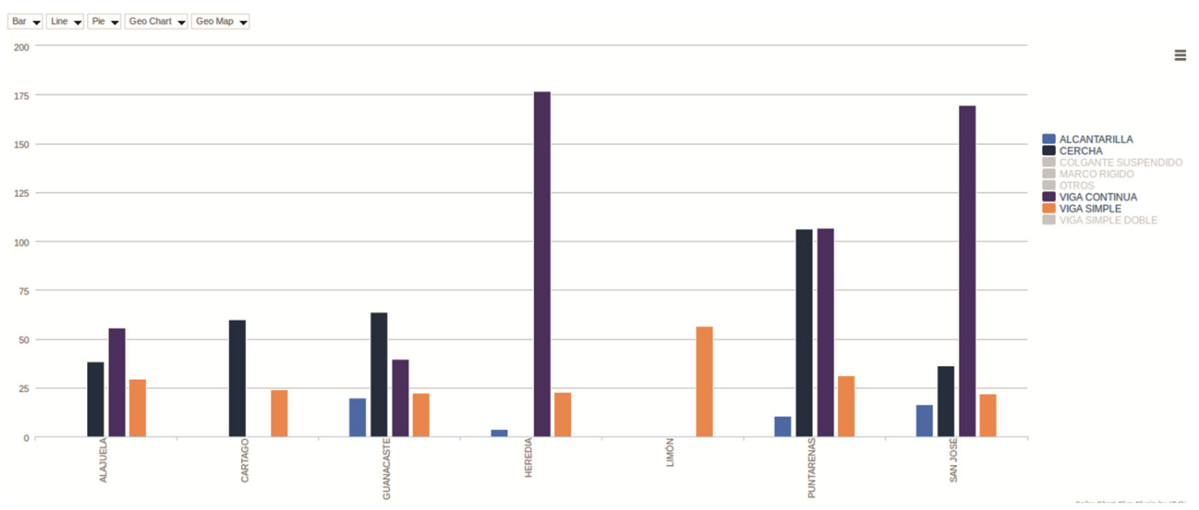

Fig. 1. Example of graphical visualization of query results.

\section{Conclusions and Future Work}

The on-going e-Bridge 3.0 project approach will allow a flexible execution of strategic queries about the real structural condition of bridges in Costa Rica through the application of business intelligence techniques such as key performance indicators panels associated to specific structural and environmental variables. These indicators and monitoring panels would have a positive impact on the efficiency of resource investment and planning of national public infrastructure. Preliminary results will be extended to include around 400 bridges and more data cubes and visualization features will be made available through a web interface in the near future. This information will be useful for a variety of audiences and organizations related to road maintenance activities including: municipalities, road contractor companies, government units, construction engineering researchers, and universities among others.

Acknowledgements. The authors wish to acknowledge the valuable contributions from all colleagues of the e-Bridge 3.0 project.

\section{References}

1. Ortiz, G., Garita, C.: The e-bridge 2.0 approach for SHM of bridges in Costa Rica. Presented at the 10th International Workshop on Structural Health Monitoring - IWSHM 2015. University of Stanford, California (2015)

2. Garita, C., Ortiz, G.: Towards a workflow management approach for health monitoring of bridges. In: Camarinha-Matos, L.M., Afsarmanesh, H. (eds.) Collaborative Systems for Smart Networked Environments. IFIP AICT, vol. 434, pp. 489-497. Springer, Heidelberg (2014)

3. Obando, A., Garita, C.: Diseño General de una Red Inalámbrica para Monitoreo de Salud de Puentes. Presented at the XXXIV Convención IEEE de Estudiantes de Centroamérica y Panamá - CONESCAPAN XXXIV, San Salvador, El Salvador (2015)

4. Gastineau, A., Johnson, T., Schultz, A.: Bridge Health Monitoring and Inspections - A Survey of Methods. University of Minnesota MN/RC 2009-29 (2009) 
5. Dong, Y., Song, R.: Bridges Structural Health Monitoring and Deterioration Detection Synthesis of Knowledge and Technology. University of Alaska Fairbanks MN/RC 2009-29 (2010)

6. Garita, C.: Enfoques de Integración de Información para Sistemas de Monitoreo de Salud Estructural de Puentes. Tecnología en Marcha, vol. 29 (2016)

7. Wenzel, H., Furtner, P., Clifton, R.: The role of structural health monitoring in the life-cyclemanagement of bridges. Presented at the 8th International Cable Supported Bridge Operators Conference - ICSBOC 2013, Edinburgh, Scotland (2013)

8. Daniele Zonta, P.E., Pozzi, M., Molignoni, M., Zandonini, R.: Monitoring load redistribution in a cable-stayed bridge. Presented at the 5th European Conference on Structural Control EACS, Genova, Italy (2012)

9. Koo, K.Y., Battista, N.D.: SHM data management system using MySQL database with MATLAB and web interfaces. Presented at the 5th International Conference on Structural Health Monitoring of Intelligent Infrastructure (SHMII-5), Cancún, México (2011)

10. Tas, N.C., Raileanu, C., Dejori, M., Neubauer, C.: Bridge sensor mart: a flexible and scalable data storage and analysis framework for structural health monitoring. Presented at the 5th International Conference on Bridge Maintenance, Safety and Management, Philadelphia, USA (2010)

11. Sonnleitner, E., Kosorus, H., Anderlik, S., Stumptner, R., Freudenthaler, B., Allmer, H., Kung, J.: Integration of data mining operations for structural health monitoring. Presented at the 8th International Workshop on Structural Health Monitoring, California, USA (2011)

12. Garita, C.: A case study of VO education in Costa Rica. In: Camarinha-Matos, L.M. (ed.) Virtual Enterprises and Collaborative Networks. IFIP, vol. 149, pp. 589-596. Springer, US (2004)

13. Garita, C., Afsarmanesh, H., Hertzberger, L.O.: A survey of distributed information management approaches for virtual enterprise infrastructures. In: Franke, U.J. (ed.) Managing Virtual Web Organizations in the 21st Century: Issues and Challenges, pp. 164-183. Idea Group Publishing (2002)

14. Afsarmanesh, H., Garita, C., Camarinha-Matos, L.M., Lima, C.: Workflow support for management of information in PRODNET II. In: 5th International Workshop on Intelligent Manufacturing Systems - IMS, Gramado, Brazil, pp. 49-54 (1998)

15. Hatle, S., Sayeed, A., Gupta, D., Neela, G., Winter, S.: Pentaho Data Integration Tool (2013) 